\title{
Preference evolution with deceptive interactions and heterogeneous trust in bounded confidence model: A simulation analysis
}

Yucheng Dong ${ }^{\mathrm{a}}$, Yuxiang Fan ${ }^{\mathrm{a}}$, Haiming Liang ${ }^{\mathrm{a},{ }^{*},}$, Francisco Chiclana ${ }^{\mathrm{b}}$, Enrique Herrera-Viedma a. Business School, Sichuan University, Chengdu 610065, China

b. Institute of Artificial Intelligence, De Montfort University, Leicester, UK

c. Department of Computer Science and Artificial Intelligence, University of Granada,

$$
\text { Granada 18071, Spain }
$$

\begin{abstract}
The bounded confidence model is a popular tool to model the evolution of preferences and knowledge in opinion dynamics. In the bounded confidence model, it is assumed that all agents are honest to express their preferences and knowledge. However, in real-life opinion dynamics, agents often hide their true preferences, and express different preferences to different people. In this paper, we propose the evolution of preferences with deceptive interactions and heterogeneous trust in bounded confidence model, in which some agents will express three types of preferences: true preferences, communicated preferences and public preferences. In the proposed model, the communication regimes of the agents are established. Based on the established communication regimes, the true preferences, communicated preferences and public preferences of the agents are updated. Furthermore, we use an agent-based simulation to unfold the influences of the deceptive interactions and heterogeneous trust on the evolutions of preferences.
\end{abstract}

Keywords: Preference evolution; Bounded confidence model; Deceptive interaction; Heterogeneous trust; Communicated preference

\section{Introduction}

In real life, people often express their preferences and knowledge over the economic, social and political issues [1-12]. Their preferences are always evolved due to the interactions among

\footnotetext{
*Corresponding author

E-mail addresses: ycdong@scu.edu.cn (Y. Dong), yfan16@buaa.edu.cn (Y. Fan), hmliang@scu.edu.cn (H. Liang), chiclana@dmu.ac.uk (F. Chiclana),viedma@decsai.ugr.es (E. Herrera Viedma)
} 
different people [13]. Opinion dynamics provides a well-established basis for describing the evolutions of preferences among individuals and for determining the finalized preference states, with many specific opinion dynamics models already developed [14-16]. Based on the representation of preference and knowledge with discrete or continuous values, the existing opinion dynamics models can be roughly divided into the respective classes of discrete and continuous models. Discrete opinion dynamics model mainly includes the voter model [17-18], the Snajzd model [19], the Ising model [20], the social impact model [21], the Axelrod Culture model [22], the Rumors model [23-24]; while continuous opinion dynamics models include the DG (DeGroot) model [25-27], and the bounded confidence model [28-29].

Among the existing opinion dynamics models, the bounded confidence models and their modified extensions have been widely applied in exploring public opinion formation and social contagious behaviors [30-31]. In the bounded confidence model, it is assumed that the agents only influence each other if the difference among their opinions is not larger than abounded confidence threshold. There exist two classical bounded confidence models: the Deffuant-Weisbuch (DW) model $[28,32]$ and the Hegselmann-Krause (HK) model [29]. Both models use the concept of bounded confidences, but differ in how many other agents each agent can interact with at a certain time. Based on the DW model and the HK model, scholars have developed some interesting extensions, such as the opinion dynamics model in the social networks [33-34], the noise bounded confidence model [35-36], the consensus reaching in the bounded confidence model [37-38], the bounded confidence model with different communication regimes [39-40], and other extended bounded confidence models [41].

Prior studies on the bounded confidence framework have made significant contributions, and they are useful to analyze real-life problems in opinion dynamics but there still exist some gaps in the bounded confidence model that must be filled because

(i) In the existing bounded confidence models, each agent expresses his/her preference and knowledge honestly. However, in real-life opinion dynamics agents often hide their true preferences and express different preferences to different people [42-48].In particular, rapid expansion of online social media (e.g., Wechat) increases the probability of deceptive/dishonest interactions.

(ii) The existing bounded confidence studies assume that each agent completely trusts other 
agents' preferences, an assumption that is sounded when all agents are honest. However, as mentioned in (i), some agents may be dishonest, and an agent might not completely trust the opinions that other agents express to him/her, and we argue that the trust level among agents has a key role to play in the bounded confidence model.

Hence, the main aim of this paper is to investigate the preference evolution with deceptive interactions and heterogeneous trust in bounded confidence model, and the influences of the deceptive interactions and heterogeneous trust on the HK bounded confidence model.

The proposal can be applied to deal with some real world problems such as, when the government attempts to publish a proposition on the gay marriage, it is necessary to investigate the dynamics of people's preferences on whether the marriage should be defined between man and woman. In this situation, people were asked to express their preferences in public, i.e., public preference. However, to safeguard the privacy, people's public preferences don't imply their true preferences. Furthermore, based on the heterogeneous trust, each individual will express different preferences (i.e., communicated preferences) to different people. Since the proposal is incorporated into the deceptive interactions and heterogeneous trust, it can provide the decision support for the government to investigate preference evolutions of people.

The rest of this paper is organized as follows: Section 2 introduces the classical HK bounded confidence model. Then, based on the studies by Castellano et al. [33] and Gross and Blasius [49], the bounded confidence model with deceptive interactions and heterogeneous trust in opinion dynamics is proposed in Section 3. In Section 4, simulation experiments are designed to investigate the influences of deceptive interactions and heterogeneous trust in the HK bounded confidence model on the evolutions of preferences. Finally, Section 5 summarizes and highlights the major contributions of this paper.

\section{The HK bounded confidence model}

In this section, we introduce the HK bounded confidence model in brief, which is also the fundamental model of our study. The DW model and the HK model both rely on the idea of repeated averaging under bounded confidence. They differ in their interaction regime. In the DW model, agents meet in random pairwise encounters after which they compromise or not. In the HK model, each agent moves to the average preference of all agents which lie in his/her area of confidence. Thus, when using the DW model as the basis of our study, a similar work can be 
run.

Let $A=\left\{A_{1}, A_{2}, \ldots, A_{N}\right\}$ be a set of agents and $t$ be a discrete time, respectively. Let $X^{\prime}(t)=\left(x_{1}^{\prime}(t), x_{2}^{\prime}(t), \ldots, x_{N}^{\prime}(t)\right)^{T}$ be the preference profile at time $t$ of the set of agents, where $x_{i}^{\prime}(t) \in[0,1]$ denotes the preference expressed by agent $A_{i} \in A$ at time $t$. Let $\varepsilon$ be the bounded confidence of the agents.

Let $I^{\prime}\left(A_{i}, X^{\prime}(t)\right)$ be the confidence set of agent $A_{i}$ at time $t$, and let $w_{i j}^{\prime}(t)$ be the weight that agent $A_{i}$ assigns to agent $A_{j}$ at time $t$, i.e.,

$$
w_{i j}^{\prime}(t)=\left\{\begin{array}{ll}
\frac{1}{\# I^{\prime}\left(A_{i}, X^{\prime}(t)\right)}, & A_{j} \in I^{\prime}\left(A_{i}, X^{\prime}(t)\right) \\
0, & A_{j} \notin I^{\prime}\left(A_{i}, X^{\prime}(t)\right)
\end{array}, \quad i=1,2, \ldots, N,\right.
$$

where $I^{\prime}\left(A_{i}, X^{\prime}(t)\right)=\left\{A_{j} \| x_{i}^{\prime}(t)-x_{j}^{\prime}(t) \mid \leq \varepsilon\right\}$, and $\# I^{\prime}\left(A_{i}, X^{\prime}(t)\right)$ is the number of agents in the set $I^{\prime}\left(A_{i}, X^{\prime}(t)\right)$. An agent $A_{i}$ only takes into account the agents $A_{j}$ in $I^{\prime}\left(A_{i}, X^{\prime}(t)\right)$. The preference $x_{i}^{\prime}(t+1)$ is formed as follows:

$$
x_{i}^{\prime}(t+1)=\sum_{A_{j} \in I^{\prime}\left(A_{i}, X^{\prime}(t)\right)} w_{i j}^{\prime}(t) x_{j}^{\prime}(t), \quad i=1,2, \ldots, N
$$

\section{The proposed model}

In this section, based on the HK model, we propose the preference evolution with deceptive interactions and heterogeneous trust in bounded confidence model. Similarly to the HK model in Section 2, let $A=\left\{A_{1}, A_{2}, \ldots, A_{N}\right\}$ be a set of agents, $t$ be a discrete time, and $\varepsilon$ be the bounded confidence level.

\subsection{Four types of preferences}

Motivated by the study of Barrio et al. (2015) [46], in the proposed opinion dynamics each agent will have four types of preferences: true preference, communicated preference, public preference and estimated true preference. Each agent will express four types of preferences within a numerical interval $[0,1]$, where ' 0 'signifies the completely positive opinion on the issue, and ' 1 'signifies the completely negative opinion on the issue. The smaller preference value indicates the agents will provide more positive opinion. For example, when the government wants to 
publish a new policy, the citizens can use the value within the interval $[0,1]$ to express "support" or "opposition" on the policy.

(1) True Preference

Let $x_{i}(t) \in[0,1]$ be the true preference of $A_{i}$ at time $t$, for $i=1,2, \ldots, N$. We assume that $x_{i}(t)$ is only known by agent $A_{i}$.

(2) Communicated preference

Since each agent has different trust levels with respect to different agents, he/she will express different preferences to different agents (i.e., deceptive interactions). We call these opinions the communicated preferences. Let $z_{i j}(t) \in[0,1]$ be the communicated preferences that the agent $A_{i}$ expresses to agent $A_{j}$ at time $t$, for $i, j=1,2, \ldots, N$ and $i \neq j$.Generally, the larger trust level that $A_{i}$ assigns to $A_{j}$, the closer the communicated preferences $z_{i j}(t)$ is to the true preference $x_{i}(t)$. We assume $z_{i j}(t)$ is only known by agents $A_{i}$ and $A_{j}$.

(3) Public Preference

Each agent will disclose a preference to all the other agents, and we call it the public preference. Let $y_{i}(t)$ denotes the public preference of $A_{i}$ at time $t$, for $i=1,2, \ldots, N$. Based on the study by Barrio et al. (2015), $y_{i}(t)$ is determined as the average of all communicated preferences:

$$
y_{i}(t)=\frac{1}{N-1} \sum_{j=1, j \neq i}^{N} z_{i j}(t)
$$

\section{(4) Estimated True Preference}

An agent does not know the true preferences of other agents, and also often cannot completely trust the received communicated preferences from other agents. Thus, when receiving other agents' communicated preferences, he/she will estimate the true preferences of these agents. For any $A_{i}, A_{j} \in A, \quad A_{i}$ can utilize two kinds of preferences, i.e. public preference and communicated preference, to estimate the true preference of $A_{j}$. Let $f_{i j}(t)(i \neq j)$ be the estimated true preferences of $A_{j}$ from the agent $A_{i}$ at time $t$ : 


$$
f_{i j}(t)=\alpha_{i j} z_{j i}(t)+\left(1-\alpha_{i j}\right) y_{j}(t), \quad i, j=1,2, \ldots, N ; i \neq j
$$

where $\alpha_{i j} \in[0,1]$ denotes the trust level that agent $A_{i}$ assigns to agent $A_{j}$. In this paper we assume the trust levels among the agents don't evolve over time. The trust levels among the agents have great influence on the estimated true preferences. As the trust level agent $A_{i}$ assigns to $A_{j}$ increases, so does the trust agent $A_{i}$ gives to the communicated preference $z_{i j}(t)$.

\subsection{Evolution of Preferences}

The preferences $x_{i}(t+1), \quad z_{i j}(t+1)$ and $y_{i}(t+1)$ are formed as follows:

Because an agent does not know the other agents' true preference, the estimated true preferences are used to estimate the confidence set of each agent. Let $I\left(A_{i}, X(t)\right)$ be the estimated confidence set of agent $A_{i}$ at time $t$, where

$$
I\left(A_{i}, X(t)\right)=\left\{A_{j}|| x_{i}(t)-f_{i j}(t) \mid \leq \varepsilon\right\}
$$

Let $w_{i j}(t)$ be the weight that $A_{i}$ assigns to $A_{j}$ at time $t, w_{i j}(t)$ is determined by

$$
w_{i j}(t)= \begin{cases}\frac{\alpha_{i j}}{\sum_{A_{j} \in I\left(A_{i}, X(t)\right)} \alpha_{i j}}, & A_{j} \in I\left(A_{i}, X(t)\right) \\ 0, & A_{j} \notin I\left(A_{i}, X(t)\right)\end{cases}
$$

In Eq. (6), the greater the value $\alpha_{i j}$, the higher the weight $w_{i j}(t)$ that $A_{i}$ assigns to $A_{j}$.

Next, let $x_{i}(t+1)$ be the true preference of agent $A_{i}$ at time $t+1$, then

$$
x_{i}(t+1)=\sum_{j=1}^{N} w_{i j}(t) f_{i j}(t)
$$

Let $z_{i j}(t+1)$ be the communicated preference that agent $A_{i}$ expresses to agent $A_{j}$ at time $t+1$, where

$$
z_{i j}(t+1)= \begin{cases}\alpha_{i j} x_{i}(t)+\left(1-\alpha_{i j}\right) z_{i j}(t) & A_{j} \in I\left(A_{i}, X(t)\right) \\ \frac{x_{i}(t+1)}{x_{i}(t)} z_{i j}(t) & A_{j} \notin I\left(A_{i}, X(t)\right)\end{cases}
$$

In Eq. (8), when $A_{j} \in I\left(A_{i}, X(t)\right), A_{i}$ will update the communicated preference $z_{i j}(t+1)$ between the previous true preference $x_{i}(t)$ and the previous communicated 
preference $z_{i j}(t)$, with high values of $\alpha_{i j}$ indicating that the agent $A_{i}$ will be more willing to express his/her true preference to $A_{j}$ at time $t+1$. Otherwise, $A_{i}$ will not reveal the information of his true thought to $A_{j}$, and thus we assume that he will keep the same ratio of his communicated preference to his true preference at the time $t+1$.

Let $y_{i}(t+1)$ be the public preference of the agent $A_{i}$ at time $t+1$. Following Eq. (3), we have

$$
y_{i}(t+1)=\frac{1}{N-1} \sum_{j=1, j \neq i}^{N} z_{i j}(t+1)
$$

Note 1. When using the DW or HK model as the basic model to proceed with preference evolution, the main difference can be reflected by the estimated preferences. In the DW-based model, each agent will only estimate the true preference of a random selected agent. However, in the HK-based model, each agent will estimate the true preference of all the other agents.

In the following, we use an example to clearly illustrate the evolutions of four types of preferences.

Example 1. Assume five agents $\left\{A_{1}, A_{2}, A_{3}, A_{4}, A_{5}\right\}$ and the bounded confidence $\varepsilon=0.2$. The true preferences $x_{i}(0)$ are shown in Table 1.

Table 1 . The true preferences $x_{i}(0)$

\begin{tabular}{cccccc}
\hline$A_{i}$ & $A_{1}$ & $A_{2}$ & $A_{3}$ & $A_{4}$ & $A_{5}$ \\
\hline$x_{i}(0)$ & 0.11 & 0.16 & 0.39 & 0.64 & 0.67 \\
\hline
\end{tabular}

The communicated preferences $z_{i j}(0)$ are shown in Table 2.

Table 2. The communicated preferences $z_{i j}(0)$

\begin{tabular}{cccccc}
\hline & $A_{1}$ & $A_{2}$ & $A_{3}$ & $A_{4}$ & $A_{5}$ \\
\hline$A_{1}$ & - & 0.15 & 0.22 & 0.07 & 0.34 \\
\hline$A_{2}$ & 0.19 & - & 0.34 & 0.25 & 0.42 \\
\hline$A_{3}$ & 0.32 & 0.21 & - & 0.38 & 0.49 \\
\hline$A_{4}$ & 0.51 & 0.62 & 0.34 & - & 0.43 \\
\hline$A_{5}$ & 0.35 & 0.67 & 0.78 & 0.44 & - \\
\hline
\end{tabular}

The public preferences $y_{i}(0)$ are shown in Table 3 . 
Table 3. The public preferences $y_{i}(0)$

\begin{tabular}{clllll}
\hline$A_{i}$ & $A_{1}$ & $A_{2}$ & $A_{3}$ & $A_{4}$ & $A_{5}$ \\
\hline$y_{i}(0)$ & 0.22 & 0.24 & 0.45 & 0.54 & 0.60 \\
\hline
\end{tabular}

The trust levels $\alpha_{i j}(i, j=1,2, \ldots, 5)$ among the agents are listed in Table 4.

Table 4. The trust levels $\alpha_{i j}$

\begin{tabular}{cccccc}
\hline & $A_{1}$ & $A_{2}$ & $A_{3}$ & $A_{4}$ & $A_{5}$ \\
\hline$A_{1}$ & - & 0.84 & 0.65 & 0.85 & 0.43 \\
\hline$A_{2}$ & 0.85 & - & 0.54 & 0.71 & 0.32 \\
\hline$A_{3}$ & 0.84 & 0.61 & - & 0.97 & 0.76 \\
\hline$A_{4}$ & 0.69 & 0.92 & 0.37 & - & 0.49 \\
\hline$A_{5}$ & 0.47 & 1 & 0.76 & 0.52 & - \\
\hline
\end{tabular}

Then, based on Eq. (4), the estimated true preferences $f_{i j}(0)$ are calculated in Table 5.

Table 5. The estimated true preferences $f_{i j}(0)$

\begin{tabular}{cccccc}
\hline & $A_{1}$ & $A_{2}$ & $A_{3}$ & $A_{4}$ & $A_{5}$ \\
\hline$A_{1}$ & - & 0.198 & 0.366 & 0.515 & 0.493 \\
\hline$A_{2}$ & 0.161 & - & 0.320 & 0.597 & 0.622 \\
\hline$A_{3}$ & 0.220 & 0.301 & - & 0.346 & 0.737 \\
\hline$A_{4}$ & 0.117 & 0.249 & 0.424 & - & 0.522 \\
\hline$A_{5}$ & 0.276 & 0.420 & 0.480 & 0.483 & - \\
\hline
\end{tabular}

Based on Eqs. (5)-(6), the weights $w_{i j}(0)$ are calculated in Table 6.

Table 6. The weights $w_{i j}(0)$

\begin{tabular}{cccccc}
\hline & $A_{1}$ & $A_{2}$ & $A_{3}$ & $A_{4}$ & $A_{5}$ \\
\hline$A_{1}$ & - & 1 & 0 & 0 & 0 \\
\hline$A_{2}$ & 0.612 & - & 0.388 & 0 & 0 \\
\hline$A_{3}$ & 0.350 & 0.251 & - & 0.399 & 0 \\
\hline$A_{4}$ & 0 & 0 & 0 & - & 1 \\
\hline$A_{5}$ & 0 & 0 & 0.594 & 0.406 & 0 \\
\hline
\end{tabular}

Furthermore, based on Eq. (7), the true preferences $x_{i}(1)$ are calculated in Table 7. 
Table 7. The true preferences $x_{i}(1)$

\begin{tabular}{cccccc}
\hline$A_{i}$ & $A_{1}$ & $A_{2}$ & $A_{3}$ & $A_{4}$ & $A_{5}$ \\
\hline$x_{i}(1)$ & 0.198 & 0.223 & 0.291 & 0.522 & 0.481 \\
\hline
\end{tabular}

Based on Eq. (8), the communicated preferences $z_{i j}(1)$ are calculated in Table 8.

Table 8 . The communicated preferences $z_{i j}(1)$

\begin{tabular}{cccccc}
\hline & $A_{1}$ & $A_{2}$ & $A_{3}$ & $A_{4}$ & $A_{5}$ \\
\hline$A_{1}$ & - & 0.112 & 0.396 & 0.126 & 0.612 \\
\hline$A_{2}$ & 0.165 & - & 0.243 & 0.348 & 0.584 \\
\hline$A_{3}$ & 0.379 & 0.320 & - & 0.390 & 0.366 \\
\hline$A_{4}$ & 0.416 & 0.505 & 0.277 & - & 0.533 \\
\hline$A_{5}$ & 0.252 & 0.481 & 0.696 & 0.560 & - \\
\hline
\end{tabular}

Based on Eq. (9), the public preferences $y_{i}(1)$ are calculated in Table 9.

Table 9. The public preferences $y_{i}(1)$

\begin{tabular}{cccccc}
\hline$A_{i}$ & $A_{1}$ & $A_{2}$ & $A_{3}$ & $A_{4}$ & $A_{5}$ \\
\hline$y_{i}(1)$ & 0.313 & 0.335 & 0.363 & 0.433 & 0.497 \\
\hline
\end{tabular}

Based on Eq. (4), the estimated true preferences $f_{i j}(1)$ are calculated in Table 10.

Table 10. The estimated true preferences $f_{i j}(1)$

\begin{tabular}{cccccc}
\hline & $A_{1}$ & $A_{2}$ & $A_{3}$ & $A_{4}$ & $A_{5}$ \\
\hline$A_{1}$ & - & 0.192 & 0.373 & 0.418 & 0.392 \\
\hline$A_{2}$ & 0.146 & - & 0.340 & 0.484 & 0.492 \\
\hline$A_{3}$ & 0.383 & 0.279 & - & 0.282 & 0.649 \\
\hline$A_{4}$ & 0.184 & 0.347 & 0.373 & - & 0.528 \\
\hline$A_{5}$ & 0.453 & 0.584 & 0.365 & 0.485 & - \\
\hline
\end{tabular}

\section{The influences of the deceptive interactions and heterogeneous trust}

In this section, we investigate the influences of the deceptive interactions and heterogeneous trust in the HK bounded confidence model, and we select the following four indexes in the simulation investigation:

(1) The stabilization time ( $S T$ ). The stabilization time refers to the time that the opinions of all the agents reach a stable state. In the simulation, we consider two kinds of stabilization time: 
Let $S T_{x}$ and $S T_{y}$ be the stabilization time for the public preferences and the true preferences, respectively. If there exists time $t_{1}$, such that $\left|x_{i}\left(t_{1}\right)-x_{i}\left(t_{1}-1\right)\right| \leq 0.001$ for each agent $A_{i}$, then we consider that the true preferences of all the agents reach a stable state at time $t_{1}$, i.e., $S T_{x}=t_{1}$. Meanwhile, if there exists time $t_{2}$, such that $\left|y_{i}\left(t_{2}\right)-y_{i}\left(t_{2}-1\right)\right| \leq 0.001$ for each agent $A_{i}$, we consider that the public preferences of all the agents reach a stable state at time $t_{2}$, i.e., $S T_{y}=t_{2}$.

(2) The number of clusters ( $N C$ ). A cluster refers to the set of agents whose preferences have no difference, and in our simulation, we assume that there is no difference if the difference between two preferences is smaller than 0.001 . We denote by $N C_{x}$ and $N C_{y}$ the number of clusters for the true preferences and the public preferences in a stable state, respectively.

(3) The correct rate of estimated confidence sets $(C R E)$. Let $t^{*}$ be the time for the true preferences of agents reaching the stable state, i.e., $t^{*}=t_{1}$, and let $f_{i j}\left(t^{*}\right)(i \neq j)$ be the estimated true preferences of $A_{j}$ from $A_{i}$ at time $t^{*}$. For any two agents $A_{i}$ and $A_{j}$, if one of the following two cases is satisfied:

(a) $\left|x_{i}\left(t^{*}\right)-x_{j}\left(t^{*}\right)\right| \leq \varepsilon$ and $\left|x_{i}\left(t^{*}\right)-f_{i j}\left(t^{*}\right)\right| \leq \varepsilon$;

(b) $\left|x_{i}\left(t^{*}\right)-x_{j}\left(t^{*}\right)\right|>\varepsilon$ and $\left|x_{i}\left(t^{*}\right)-f_{i j}\left(t^{*}\right)\right|>\varepsilon$,

then $A_{i}$ makes the right estimation for $A_{j}$ being or not in his/her confidence set at time $t^{*}$.

Let $r e_{i}\left(t^{*}\right)$ be the set of agents with right estimation made by $A_{i}$, then $r e_{i}\left(t^{*}\right)=\left\{A_{j} \mid\right.$ $\left|x_{i}\left(t^{*}\right)-x_{j}\left(t^{*}\right)\right| \leq \varepsilon$ and $\left.\left|x_{i}\left(t^{*}\right)-f_{i j}\left(t^{*}\right)\right| \leq \varepsilon\right\} \bigcup\left\{\left|x_{i}\left(t^{*}\right)-x_{j}\left(t^{*}\right)\right|>\varepsilon\right.$ and $\left.\left|x_{i}\left(t^{*}\right)-f_{i j}\left(t^{*}\right)\right|>\varepsilon\right\}$, and let $\# r e_{i}\left(t^{*}\right)$ be its cardinality. If $C R E_{i}\left(t^{*}\right)=\frac{\# r e_{i}\left(t^{*}\right)}{N-1}$, let $C R E$ be the average $C R E_{i}\left(t^{*}\right)$ value of all the agents, i.e., $C R E=\frac{\sum_{i=1}^{N} C R E_{i}\left(t^{*}\right)}{N}$.

(4) The size of the lies (SL). Let $S L_{i}\left(t^{*}\right)=\frac{\sum_{j}\left|x_{i}\left(t^{*}\right)-z_{i j}\left(t^{*}\right)\right|}{N-1}$ be the average 
differences between the true preference and all communicated preferences that agent $A_{i}$ expresses, which is used to measure the size of the lies of such agent $A_{i}$ at stable state time $t^{*}$. Let $S L$ be the average value of all the agents $S L_{i}\left(t^{*}\right)$ values at time $t^{*}$, i.e., $S L=\frac{\sum_{i=1}^{N} S L_{i}\left(t^{*}\right)}{N}$

Next, before conducting the simulations, we define the concept of honest agent as follows: For each agent $A_{i}$, if all the communicated preferences expressed by $A_{i}$ are same to his/her true preference at any time $t$, i.e., $z_{i j}(t)=y_{i}(t)=x_{i}(t)$, then we say that agent $A_{i}$ is an honest agent. As commented in Section 3.1, each agent only know his/her own true preference. Thus, each agent is not able to judge whether other agents belongs to honest type.

Furthermore, because the effects of the bounded confidence value $\varepsilon$ in the original HK model and our proposal are similar, without loss of generality, we set $\varepsilon$ to be a fixed value of 0.15 in all simulation experiments.

\subsection{The influences of the deceptive degree of public preferences}

In our simulation, the initial true preference $x_{i}(0)(i=1,2, \ldots, N)$ is a random number sampled from the uniform distribution on the interval $[0,1]$, and the initial communicated preference $z_{i j}(0)$ is a random number sampled from the uniform distribution on the interval $\left[\max \left(0, x_{i}(0)-r\right), \min \left(1, x_{i}(0)+r\right)\right]$, where $r$ shows the difference between the public preference and the true preference. And the initial public preference is obtained as: $y_{i}(0)=\frac{1}{N-1} \sum_{j=1, j \neq i}^{N} z_{i j}(0)$. Obviously, the higher the value of $r$, the larger the difference between $x_{i}(0)$ and $y_{i}(0)$, and thus we can use $r$ to measure the deceptive degree of public preferences. Furthermore, the value of the trust level $\alpha_{i j}$ is a random number sampled from the uniform distribution on the interval $[0,1]$.

Next, we investigate the influences of the deceptive degree of public opinions $r$ and the bounded confidence $\varepsilon$ on the HK bounded confidence model.

Let $N=500$. Eqs. (3)-(9) are used to obtain the values for $S T, N C, C R E$ and $S L$ under different $r$ and $\varepsilon$ values. We run the simulation 500 times, obtaining the average values for $S T, N C, C R E$ and $S L$ under different $r$ and $\varepsilon$ values, which are shown in Figures 
$1-4$.
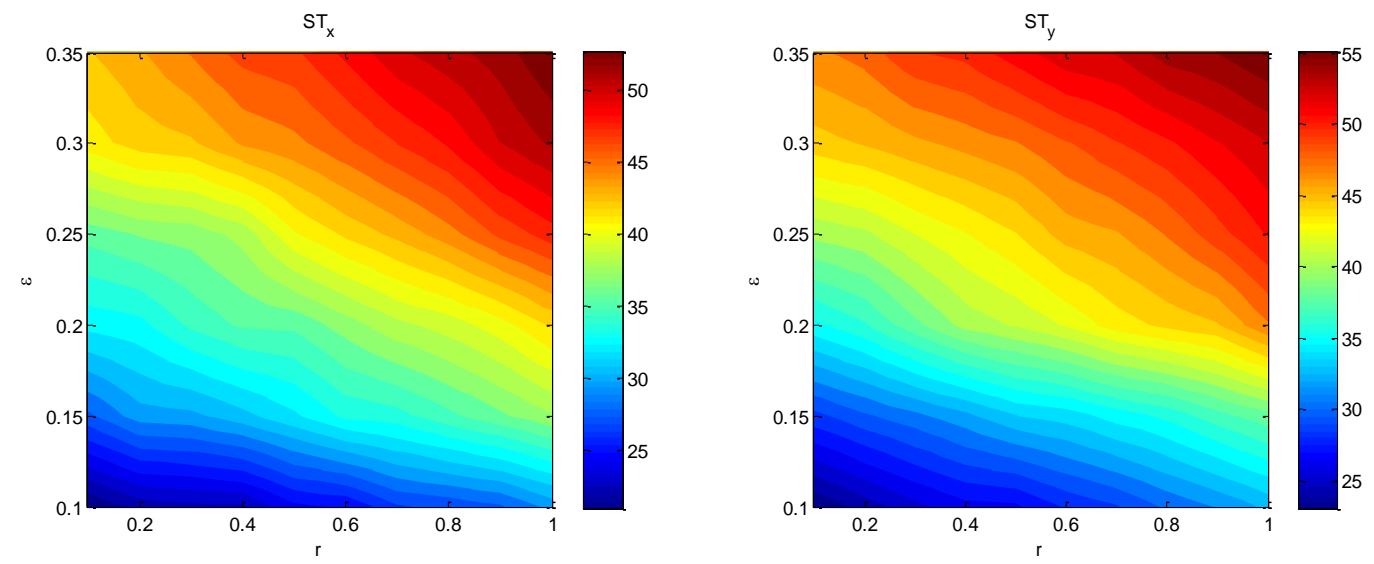

Figure 1: The $S T_{x}$ and $S T_{y}$ values under different deceptive degree of the public preferences $r$ and different bounded confidence $\varepsilon$

In Figure 1, we show the influence on the stabilization time $S T_{x}$ and $S T_{y}$ with varying values of the deceptive degree of public preferences $r$ and bounded confidence $\varepsilon$. We obtain two observations: (1) both the values of $S T_{x}$ and $S T_{y}$ increase as $r$ increases; (2) both the values of $S T_{x}$ and $S T_{y}$ increase as $\varepsilon$ increases. This implies that it will take more time for both the agents' public preferences and true preferences to reach the stable state with the increase of the deceptive degree of public preferences and bounded confidences.

The observations from Figure 1 can be explained as follows: With the increase of the deceptive degree of public preferences, the randomness of the updated preference for the agents will be strengthened, which will further lead to more time to reach the stable state. Meanwhile, the interaction with the agents who express the public preference with a larger deceptive degree will become more frequently with the increase in the bounded confidences, which will also lead to more time to reach the stable state. 

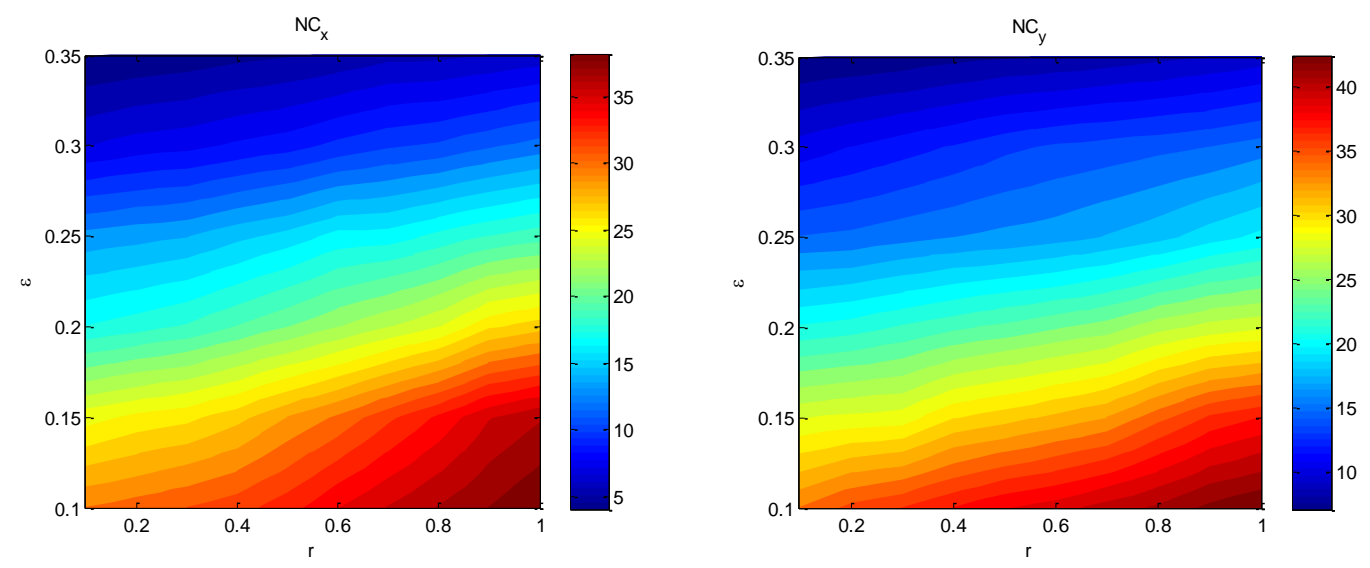

Figure 2: The $N C_{x}$ and $N C_{y}$ values under different deceptive degree of the public preferences $r$ and different bounded confidence $\varepsilon$

Figure 2 shows the number of clusters $N C_{x}$ and $N C_{y}$ with varying values of the deceptive degree of public preferences $r$ and bounded confidence $\varepsilon$. It can be seen the following two observations: (1) both $N C_{x}$ and $N C_{y}$ values increase as $r$ increases, and (2) both $N C_{x}$ and $N C_{y}$ values decrease as $\varepsilon$ increases. This implies that the number of clusters for both public preferences and true preferences will increase with the increase in the deceptive degree of public preferences. Meanwhile, the opposite result will happen with the increase of bounded confidences.

These two observations from Figure 2 can be explained as follows: As mentioned above, the randomness of the updated preference for the agents will increase with the increase of the deceptive degree of public preferences. Consequently, more clusters in the stable state will be yielded. Meanwhile, more agents will have more opportunities to communicate with each other with the increase of the bounded confidences, which will lead to less clusters in the stable state. 


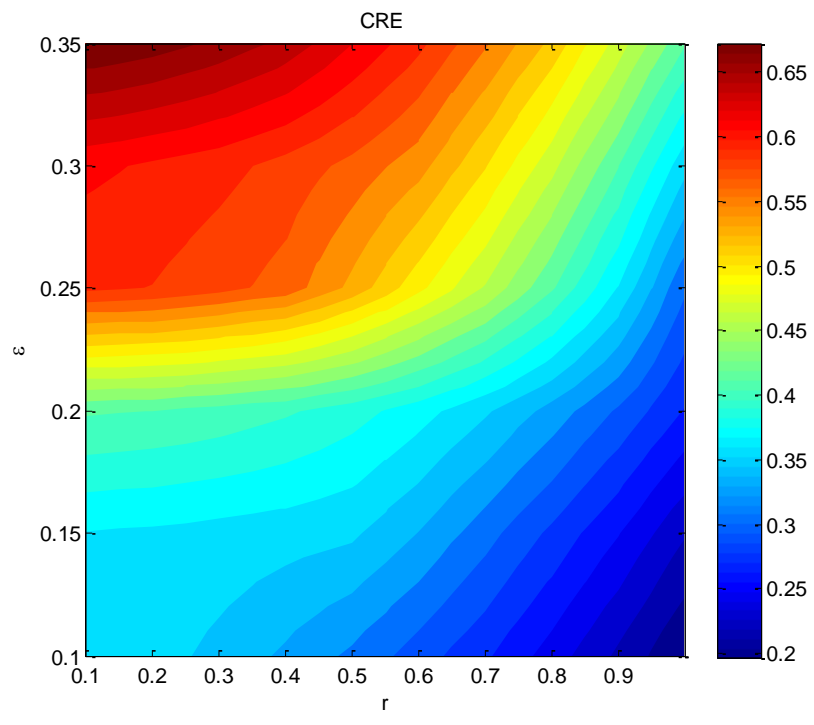

Figure 3: The CRE values under different deceptive degree of the public preferences $r$ and different bounded confidence $\varepsilon$

In Figure 3, the correct rate of estimated confidence sets with varying values of the deceptive degree of public preferences $r$ and bounded confidence $\varepsilon$ are illustrated, and the following two observations can be drawn: (1) the CRE value decreases as $r$ increases, and (2) the $C R E$ value increases as $\varepsilon$ increases.

These two observations from Figure 3 can be explained as follows: With the increase of deceptive degree of public preferences, it will become much harder for the agents to estimate the preferences with the higher accuracy. As a result, the lower correct rate of estimated confidence sets will be yielded.

Meanwhile, with the increase of the bounded confidences, the agents will have more opportunities to communicate with each other, which will further lead to the increase of the scale of the confidence sets. Consequently, this will lead to the higher correct rate of estimated confidence sets. 


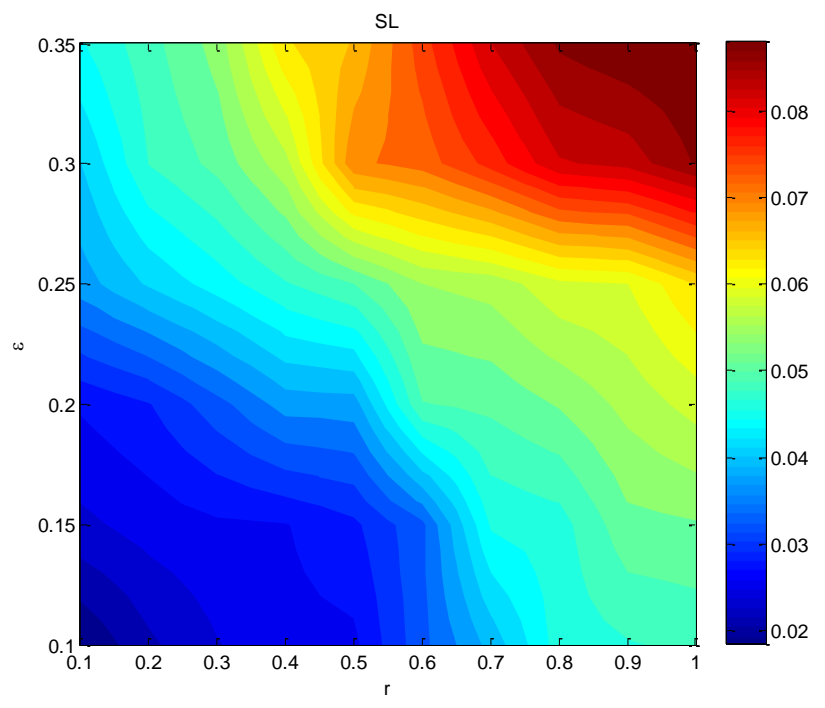

Figure 4: The $S L$ values under different deceptive degree of the public preferences $r$ and different bounded confidence $\varepsilon$

Figure 4 shows the size of the lies with varying values of the deceptive degree of public preference $r$ and bounded confidence $\varepsilon$. It is observed that: (1) the $S L$ value increases as $r$ increases, and (2) the $S L$ value increases as $\varepsilon$ increases. These observations imply that with the increase of the deceptive degree of public preferences and bounded confidences, less agents choose to express the true preferences.

These two observations from Figure 4 can be explained as follows: In the simulation, we find that the values of $\left|y_{i}\left(t^{*}\right)-x_{i}\left(t^{*}\right)\right|$ increase with the increase in the deceptive degree of public opinions and bounded confidences. Generally, large values of $\left|y_{i}\left(t^{*}\right)-x_{i}\left(t^{*}\right)\right|$ indicate that the values for $\left|z_{i j}\left(t^{*}\right)-x_{i}\left(t^{*}\right)\right|$ are large, which leads to large size of lies.

\subsection{The influence of the ratio of honest agents}

In Section 4.1 all agents are assumed to be dishonest, while in this section discusses the existence of honest agents. Let $p(0 \leq p \leq 1)$ be the proportion of agents that are honest. In the simulation, without loss of generality, it is assumed the first $p \times N$ agents $A_{i}$ $(i=1,2, \ldots, N \times p)$ are the honest agents, and the other $p \times(1-N)$ agents $A_{i}$ $(i=N \times p+1, N \times p+2, \ldots, N)$ are not. Then, for the first $p \times N$ honest agents, their initial true preferences $x_{i}(0) \quad(i=1,2, \ldots, N \times p)$ are uniformly and randomly selected from [0,1], and 
$z_{i j}(0)=y_{i}(0)=x_{i}(0)$

For the other $p \times(1-N)$ agents $A_{i}(i=N \times p+1, N \times p+2, \ldots, N)$, their initial true preferences $x_{i}(0)$, public preferences $y_{i}(0)$, and communicated preferences $z_{i j}(0)$, and the value of the trust level $\alpha_{i j}$ are generated in the same way as described in Section 4.1.

Next, let $N=100$ and $r=0.5$, the influences of the ratio of honest agents $p$ and the trust level $\varepsilon$ on the HK bounded confidence model are studied and analyzed in Figures 5-8.
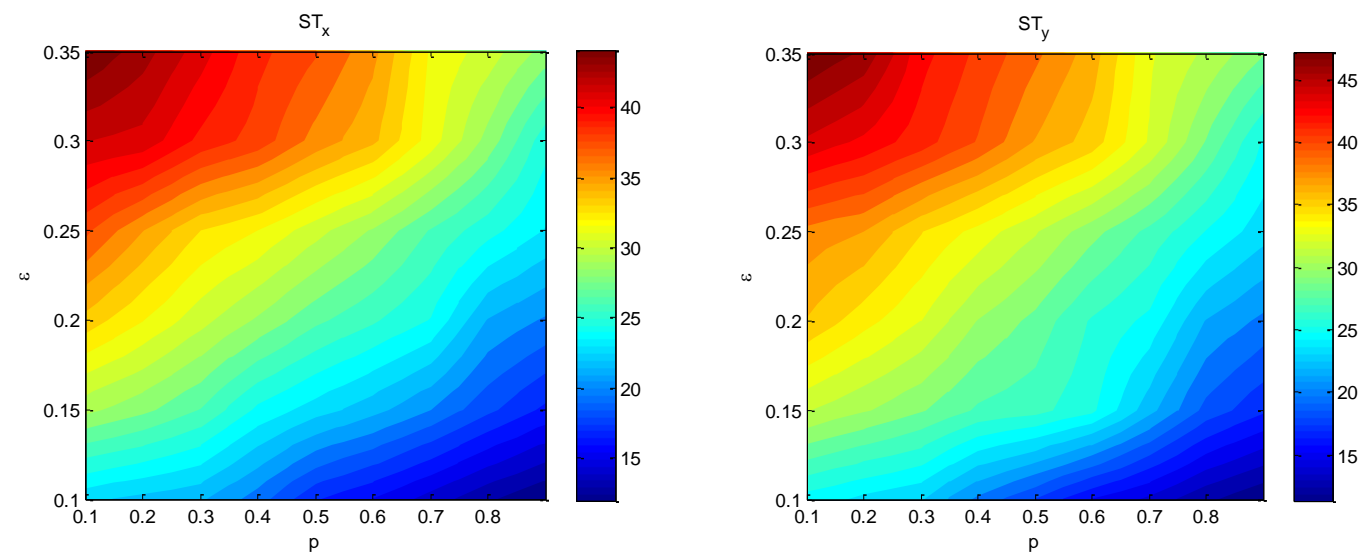

Figure 5: The $S T_{x}$ and $S T_{y}$ values under different ratio of honest agents $p$ and different bounded

Confidence $\varepsilon$

Figure 5 shows the variation of stabilization times $S T_{x}$ and $S T_{y}$ with respect to $p$ and $\varepsilon$. From Figure 5, we observe both the values of $S T_{x}$ and $S T_{y}$ decrease as $p$ increases. This implies that it takes less time for the agents to reach the stable state with the increase in the number of honest agents. This can be explained as follows: with the increase in the number of honest agents, the agents will have a lower number of other agents' preferences to estimate and so the opinions will be with smaller randomness, which would lead to less time to reach the stable state. 

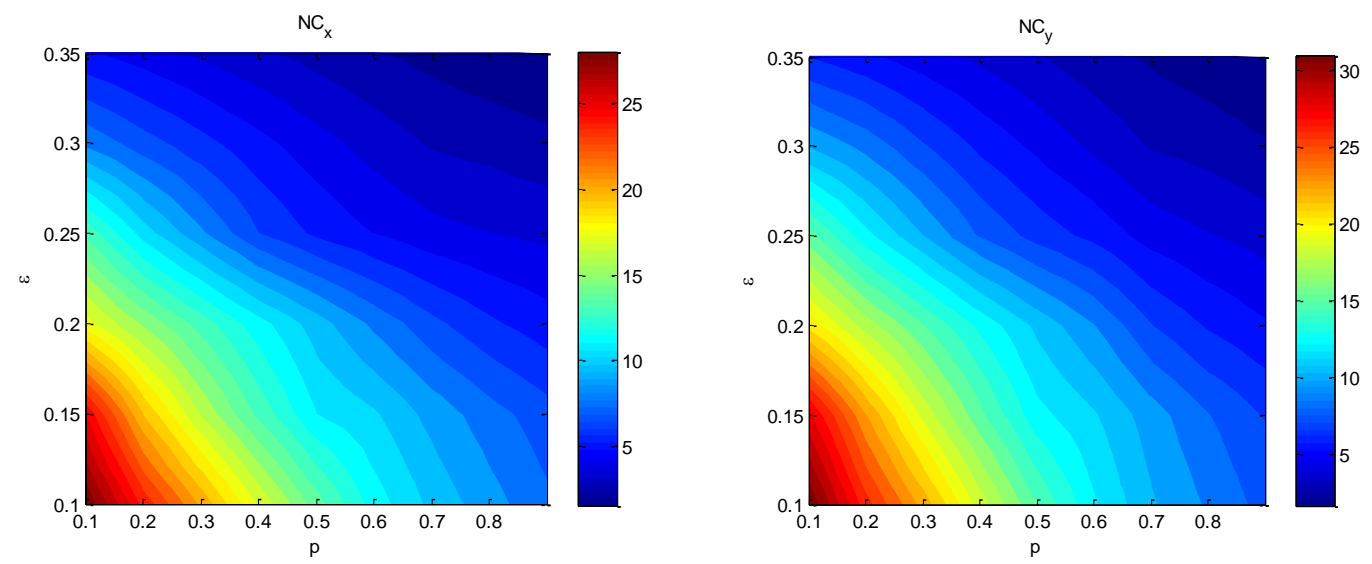

Figure 6: The $N C_{x}$ and $N C_{y}$ values under different ratio of honest agents $p$ and different bounded confidence $\varepsilon$

Figure 6 shows the variation of the number of clusters $N C_{x}$ and $N C_{y}$ with respect to $p$ and $\varepsilon$. It is observed that both $N C_{x}$ and $N C_{y}$ values decrease as $p$ increases. This implies that fewer clusters will appear with the increase in the number of honest agents, which can be explained as follows: With the increase of the number of honest agents, more agents will observe and estimate an uniform preference for the same agent, which will lead to the updated preferences with the smaller differences. Thus, fewer clusters on the opinion of agents will appear.

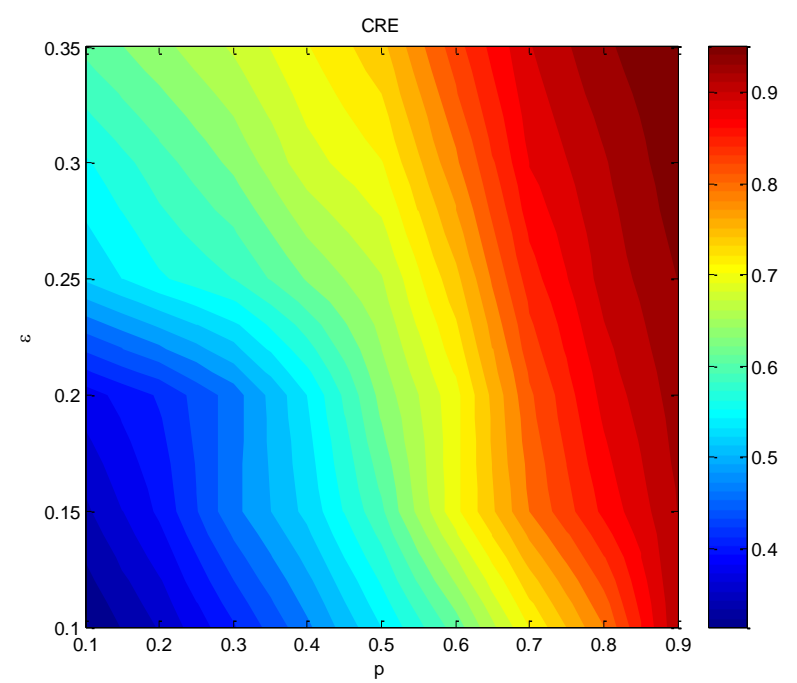

Figure 7: The $C R E$ values under different ratio of honest agents $p$ and different bounded confidence $\varepsilon$

Figure 7 shows the correct rate of estimated confidence sets with respect to $p$ and $\varepsilon$. It is observed that the $C R E$ values increase as $p$ increases. Thus, more honest agents will yield higher correct rate of estimated confidence set. This can be explained as follows: With the 
increase of the number of the honest agents, the accuracy of the estimated preferences for each agent will be strengthened. Thus, in this situation, a higher correct rate of estimated confidence sets will result.

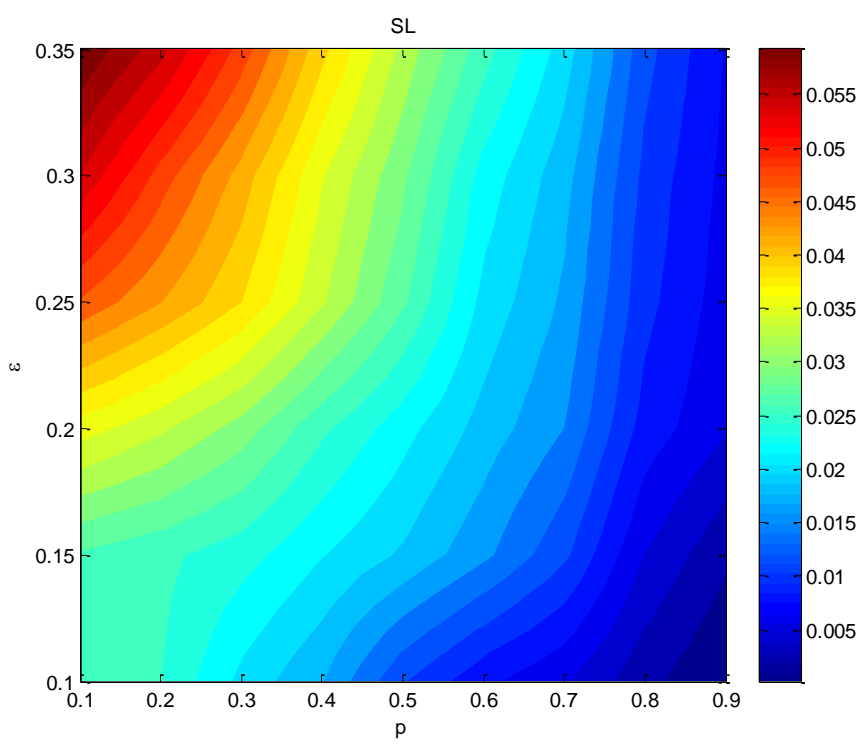

Figure 8: The $S L$ values under different ratio of honest agents $p$ and different bounded confidence $\varepsilon$

From Figure 8, which shows the size of the lies with respect to $p$ and $\varepsilon$, it is observed that the $S L$ value decreases as $p$ increases, which imply that: more agents choose to express their true preferences with the increase of the number of honest agents.

This can be explained as follows: the values of $\left|y_{i}\left(t^{*}\right)-x_{i}\left(t^{*}\right)\right|$ of non honest agents will decrease with the increase of the number of honest agents, which will further decrease the difference of the values of $\left|y_{i}\left(t^{*}\right)-x_{i}\left(t^{*}\right)\right|$. Thus, in this situation, the smaller size of lies will be yielded.

\subsection{The influence of the trust levels}

In this section, all agents are assumed to be dishonest. Their initial true preferences $x_{i}(0)$, public preferences $y_{i}(0)$, and communicated preferences $z_{i j}(0)$ are generated in the same way as described in Section 4.1. As commented in section 3.1, this paper assumes the trust levels among the agents don't evolve over time. Let $\alpha$ denote the maximum threshold for the trust levels among the agents, where $\alpha \in[0,1]$. Then, the trust level $\alpha_{i j}$ is a random number sampled from the uniform distribution on the interval $[0, \alpha]$. Obviously, the larger $\alpha$ values indicate the larger trust levels among the agents. 
Next, let $N=500$ and $r=0.5$, the influences of the trust level $\alpha$ and the bounded confidence $\varepsilon$ on the HK bounded confidence model are studied and analyzed in Figures 9-12.
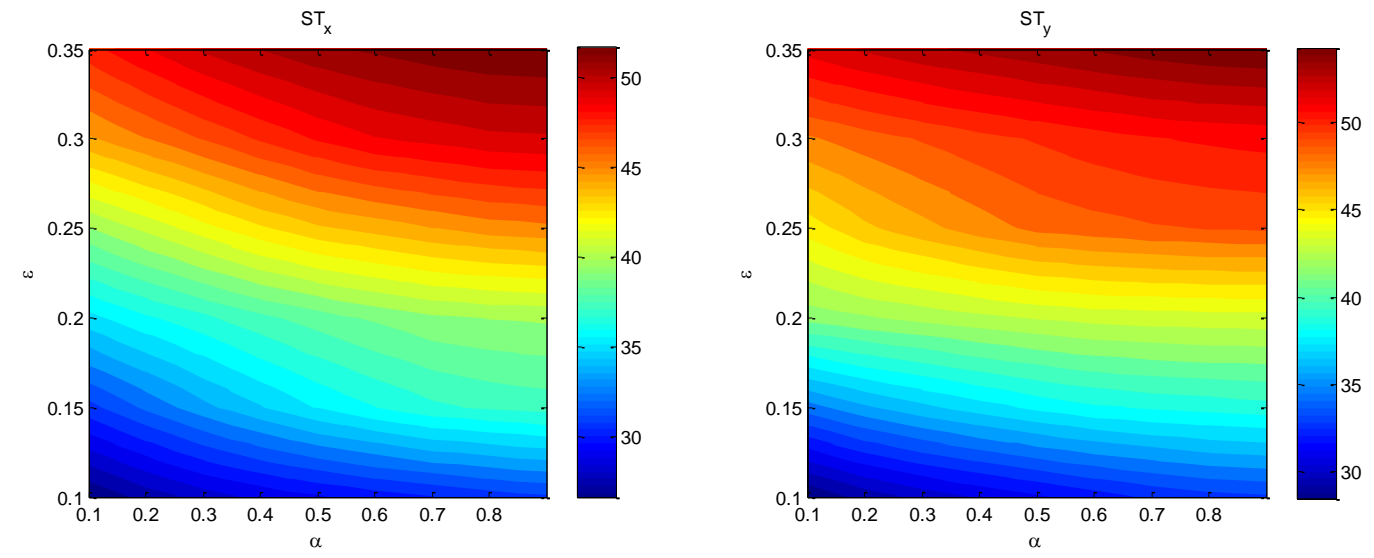

Figure 9: The $S T_{x}$ and $S T_{y}$ values under different trust level $\alpha$ and different bounded confidence $\varepsilon$

Figure 9 shows the variation of stabilization times $S T_{x}$ and $S T_{y}$ with respect to $\alpha$ and $\varepsilon$. From Figure 9, both the values of $S T_{x}$ and $S T_{y}$ increase at slow speed as $\alpha$ increases. This implies that: the increase of trust levels among the agents has weak influence on the increase of time for reaching the stable state.

This can be explained as follows: With the increase of the trust levels among the agents, the estimated preferences of the agents will be prone to the communicated preferences that the agents received, which will further lead to the difference on the estimated preferences of the same agent. Meanwhile, in the simulation we observe: When setting different trust levels, there exist the smaller differences among the estimated preferences for the same agent. Furthermore, the randomness of updated preferences will increase at slow speed. As a result, the time for reaching the stable state increases at slow speed. 

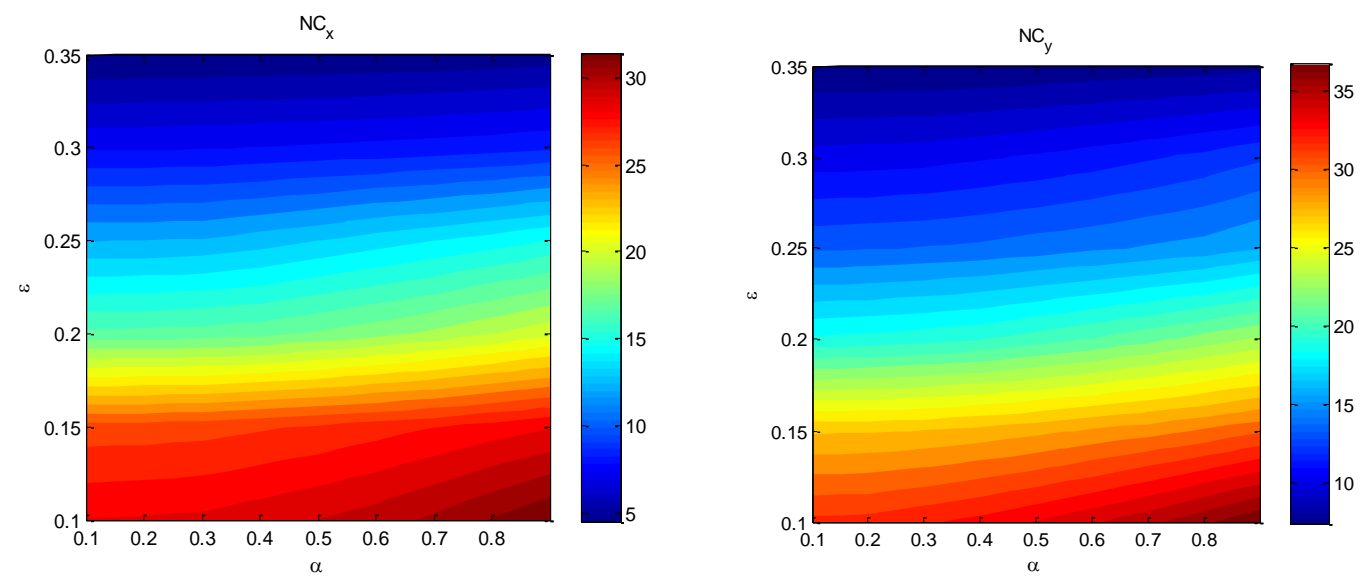

Figure 10: The $N C_{x}$ and $N C_{y}$ values under different trust level $\alpha$ and different bounded confidence $\varepsilon$

Figure 10 shows the variation of the number of clusters $N C_{x}$ and $N C_{y}$ with respect to $\alpha$ and $\varepsilon$. Both $N C_{x}$ and $N C_{y}$ values increase at slow speed as $\alpha$ increases. This implies that: the increase of trust levels among the agents has weak influence on the increase of number of clusters.

This can be explained as follows: In the simulation, we observe the differences on the estimated preferences of the same agent will increase at slow speed with the increase of the trust levels among the agents. Thus, in this situation, the number of clusters increases at slow speed.

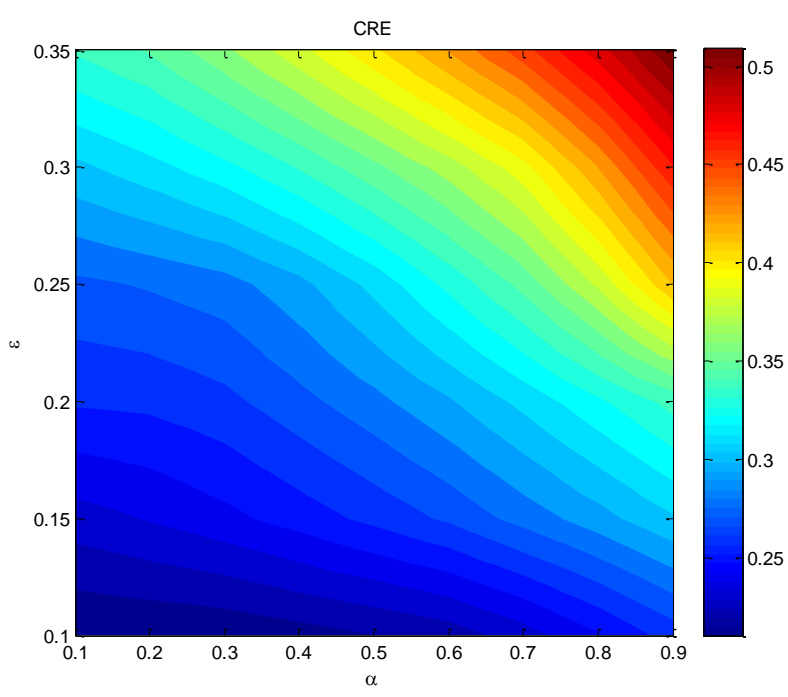

Figure 11: The $C R E$ values under different trust level $\alpha$ and different bounded confidence $\varepsilon$

Figure 11 shows the correct rate of estimated confidence sets with respect to $\alpha$ and $\varepsilon$. It is observed that the $C R E$ values increase as $\alpha$ increases. This implies that the higher correct rate of estimated confidence set will be yielded with the increase of the trust levels among the 
agents. This can be explained as follows: With the increase of the trust levels among the agents, the communicated preferences of agents will be prone to their true preferences. Consequently, a higher correct rate of estimated confidence sets will result.

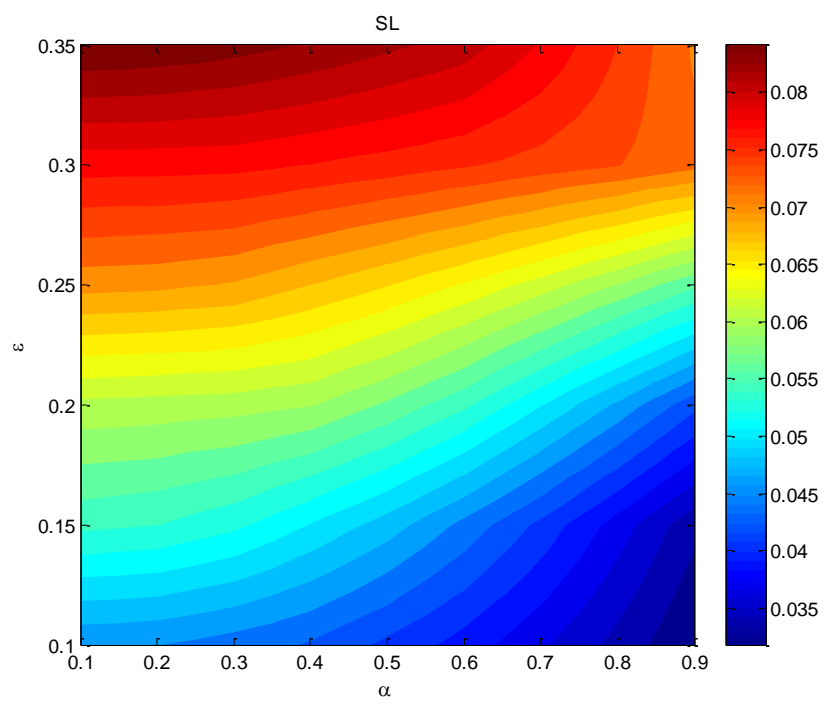

Figure 12: The $S L$ values under different trust level $\alpha$ and different bounded confidence $\varepsilon$

Figure 12 shows the size of the lies with respect to $\alpha$ and $\varepsilon$, it is observed that the $S L$ value decreases as $\alpha$ increases, which imply that: more agents choose to express their true preferences with the increase of the trust levels among the agents.

This can be explained as follows: With the increase of the trust levels among the agents, the estimated preferences of agents will be prone to the communicated preferences they received, which will lead to the smaller values of $\sum_{j}\left|x_{i}\left(t^{*}\right)-z_{i j}\left(t^{*}\right)\right|$ after the preference evolution. Thus, in this situation, the smaller size of lies will be yielded.

The observations in Sections 4.1-4.3 can be summarized as follows:

(i) With the increase of the deceptive degree of public preferences, both the agents' public preferences and true preferences will take more time to reach the stable state, more clusters for both public preferences and true preferences will appear, and the lower correct rate of estimated confidence sets and the larger size of lies will be yielded.

(ii)With the increase of the number of honest agents, both the agents' public preferences and true preferences will take less time to reach the stable state, fewer clusters for both public preferences and true preferences will appear, and the higher correct rate of estimated confidence sets and the smaller size of lies will be yielded. 
(iii)With the increase of the trust levels among the agents, the higher correct rate of estimated confidence sets and the smaller size of lies will be yielded. And the increase of the trust levels among the agents has weakly influence on the increase of time for reaching the stable state and the number of clusters.

(iv)With the increase of the bounded confidence, both the agents' public preferences and true preferences will take more time to reach the stable state, fewer clusters for both public preferences and true preferences will appear, and the higher correct rate of estimated confidence sets and the larger size of lies will be yielded.

\section{Conclusions}

In this study, we investigated the evolution of preferences with deceptive interactions and heterogeneous trust in bounded confidence model. In the proposed model, agents are assumed to be dishonest, and will express different preferences to different agents based on the trust level among them. An agent-based simulation was carried out to investigate the influences of the deceptive degree of public preferences, the group average trust and the ratio of honest agents on the bounded confidence model. It was shown that the deceptive interactions and heterogeneous trust strongly impacts the evolution of preferences.

Deceptive/dishonest interaction is a common phenomenon, and thus the proposed model can provide a good approximation to the opinions dynamic problem and, consequently, can be applied to address real opinion interaction problems. For example, when government attempts to analyze the dynamics of public opinions on a new chemical project, insurance scheme or political reform, some citizens may not express true preferences to the issue due to factors such as social pressure and lack of trust. Meanwhile, some agents may still insist on expressing their preferences to all the agents honestly. Thus, our proposal can predict the formation of public preferences to provide a better decision support for government in those cases.

Although the HK bounded confidence model is a popular tool in opinions dynamic, it is still necessary to investigate the influences of the deceptive interactions and heterogeneous trust on the formation of preferences based on the use of different opinions dynamic model (e.g., voter model and DG model etc.). On the other hand, it is necessary to investigate the social network group consensus reaching process [50-51] with consideration of deceptive interactions. 


\section{Acknowledgement}

This work was supported by the grants (No. 71571124, No. 71601133 and No. 71804148) from NSF of China, the grants (Nos. sksyl201705 and 2018hhs-58) from Sichuan University, and the grant TIN2016-75850-R supported by the Spanish Ministry of Economy and Competitiveness with FEDER funds.

\section{References}

[1] I.J. Perez, F.J. Cabrerizo, S. Alonso, Y.C. Dong, F. Chiclana, E. Herrera-Viedma. On the dynamic consensus processes in group decision making problems. Information Sciences, 459 (2018) 20-35.

[2] Y.C. Dong, Q.B. Zha, H.J. Zhang, G. Kou, H. Fujita, F. Chiclana, E. Herrera-Viedma, Consensus reaching in social network group decision making: Research paradigms and challenges. Knowledge-Based Systems, 162 (2018) 3-13.

[3] Z. Zhang, C.H. Guo, L. Martínez, Managing multigranular linguistic distribution assessments in large-scale multiattribute group decision making. IEEE Transactions on Systems, Man, and Cybernetics: Systems, 47(11) (2017) 3063-3076.

[4] C.C. Li, Y.C. Dong, F. Herrera. A consensus model for large-scale linguistic group decision making with a feedback recommendation based on clustered personalized individual semantics and opposing consensus groups. IEEE Transactions on Fuzzy Systems. Doi: 10.1109/TFUZZ.2018.2857720. (In press).

[5] H.J. Zhang, I. Palomares, Y.C. Dong, W.W. Wang, Managing non-cooperative behaviors in consensus-based multiple attribute group decision making: An approach based on social network analysis. Knowledge-Based Systems. Doi: 10.1016/j.knosys.2018.06.008. (In press).

[6] H.J. Zhang, Y.C. Dong, F. Chiclana, S. Yu. Consensus efficiency in group decision making: A comprehensive comparative study and its optimal design, European Journal of Operational Research, Doi:10.1016/j.ejor.2018.11.052 (In press).

[7] Y.T. Liu, Y.C. Dong, H.M. Liang, F. Chiclana, E. Herrera-Viedma. Multiple attribute strategic weight manipulation with minimum cost in a group decision making context with interval attribute weights information. IEEE Transactions on Systems, Man, and Cybernetics: Systems. Doi: 10.1109/TSMC.2018.2874942. (In press).

[8] H.J. Zhang, Y.C. Dong, E. Herrera-Viedma. Consensus building for the heterogeneous large-scale GDM with the individual concerns and satisfactions. IEEE Transactions on Fuzzy Systems. 26(2) (2018) 884-898.

[9] Urena R, Kou G, Dong Y, Chiclana F, Herrera-Viedma E. A review on trust propagation and opinion dynamics in social networks and group decision making frameworks. Information 
Sciences. 478 (2019) 461-475.

[10]B.W. Zhang, Y.C. Dong, E. Herrera-Viedma, Group decision making with heterogeneous preference structures: An automatic mechanism to support consensus reaching. Group Decision and Negotiation, Doi: 10.1007/s10726-018-09609-y (In press).

[11]J. Wu, F. Chiclana, H. Fujita, E. Herrera-Viedma, A visual interaction consensus model for social network group decision making with trust propagation. Knowledge-Based Systems. 122 (2017) 39-50.

[12]F .Liu, X. Li, Y. Ding, H. Zhao, X. Liu, Y. Ma, B. Tang, A social network-based trust-aware propagation model for P2P systems. Knowledge-Based Systems. 41(2013)8-15.

[13]D. Varshney, S. Kumar, V. Gupta, Predicting information diffusion probabilities in social networks: A Bayesian networks based approach. Knowledge-Based Systems, 133(2017) 66-76.

[14]Y.C. Dong, M. Zhan, G. Kou, Z.G. Ding, H.M. Liang. A survey on the fusion process in opinion dynamics. Information Fusion, 43(2018) 57-65.

[15]Z. Wang, Z. Li, G.Yuan, Y. Sun, X. Rui, X. Xiang, Tracking the evolution of overlapping communities in dynamic social networks. Knowledge-Based Systems, 157(2018) 81-97.

[16]Z.P. Tian, R.X. Nie, J.Q. Wang, H.Y. Zhang, A two-fold feedback mechanism to support consensus-reaching in social network group decision-making. Knowledge-Based Systems. Doi: 10.1016/j.knosys.2018.09.030. (In press).

[17]R.A. Holley, T.M. Liggett. Ergodic theorems for weakly interacting infinite systems and the voter model. The annals of probability. (1975) 643-663.

[18]M.J. De Oliveira. Isotropic majority-vote model on a square lattice. Journal of Statistical Physics, 66(1) (1992) 273-281.

[19]K. Sznajd-Weron, J. Sznajd. Opinion evolution in closed community. International Journal of Modern Physics C, 11(6) (2000) 1157-1165.

[20]M. Creutz, Deterministic ising dynamics. Annals of physics, 167(1)(1986) 62-72.

[21]A. Nowak, J. Szamrej, B. Latané, From private attitude to public opinion: A dynamic theory of social impact. Psychological Review. 97(3) (1990) 362.

[22]R. Axelrod, The dissemination of culture a model with local convergence and global polarization. Journal of conflict resolution, 41(2)(1997) 203-226.

[23]S. Galam, Minority opinion spreading in random geometry. The European Physical Journal B-Condensed Matter and Complex Systems. 25(4) (2002) 403-406.

[24]S. Galam. Modelling rumors: the no plane Pentagon French hoax case. Physica A: Statistical Mechanics and Its Applications, 320 (2003) 571-580.

[25]M.H. DeGroot, Reaching a consensus. Journal of the American Statistical Association. 69(345) (1974) 118-121.

[26]R.L. Berger, A necessary and sufficient condition for reaching a consensus using DeGroot's method. Journal of the American Statistical Association, 76(374) (1981) 415-418.

[27]Y.C. Dong, Z.G. Ding, L. Martínez, F. Herrera, Managing consensus based on leadership in opinion dynamics. Information Sciences, (397-398) (2017) 187-205. 
[28]G. Deffuant, D. Neau, F. Amblard, G. Weisbuch, Mixing beliefs among interacting agents. Advances in Complex Systems. 3(01n04)(2000) 87-98.

[29]R. Hegselmann, U. Krause, Opinion dynamics and bounded confidence models, analysis, and simulation. Journal of artificial societies and social simulation, 5(3)(2002).

[30]X. Chen, H.J. Zhang, Y.C. Dong. The fusion process with heterogeneous preference structures in group decision making: A survey. Information Fusion, 24 (2015) 72-83.

[31]Y.C. Dong, H.J. Zhang, E. Herrera-Viedma. Consensus reaching model in the complex and dynamic MAGDM problem. Knowledge-based Systems, 106(2016) 206-219.

[32]J. Lorenz, Continuous opinion dynamics under bounded confidence: A survey. International Journal of Modern Physics C, 18(12)(2007) 1819-1838.

[33]C. Castellano, S. Fortunato, V. Loreto. Statistical physics of social dynamics. Review of Modern Physics. 81(2) (2009) 591-646.

[34]D. Stauffer, A. Sousa, C. Schulz, Discretized opinion dynamics of the Deffuant model on scale-free networks. Journal of Artificial Societies and Social Simulation, 7(3) (2004).

[35]A. Carro, R. Toral, M. San Miguel, The role of noise and initial conditions in the asymptotic solution of a bounded confidence, continuous-opinion model. Journal of Statistical Physics, 151(1-2) (2013) 131-149.

[36]Y.Y. Zhao, L.B. Zhang, M.F. Tang, G. Kou, Bounded confidence opinion dynamics with opinion leaders and environmental noises. Computers \& Operations Research, 74(2016) 205-213.

[37]S. Fortunato, On the consensus threshold for the opinion dynamics of Krause-Hegselmann. International Journal of Modern Physics C, 16(2) (2005) 259-270.

[38]E. Wedin, P. Hegarty, The hegselmann-krause dynamics for the continuous-agent model and a regular opinion function do not always lead to consensus. IEEE Transactions on Automatic Control, 60(9)(2015) 2416-2421.

[39]L. Salzarulo, A continuous opinion dynamics model based on the principle of meta-contrast. Journal of Artificial Societies and Social Simulation, 9(1) (2006).

[40]A. Parravano, A. Andina-Díaz, M.A. Meléndez-Jiménez, Bounded confidence under preferential flip: A coupled dynamics of structural balance and opinions. PloS one, 11(10)(2016) e0164323.

[41]Y.Y. Zhao, G. Kou, Y.Peng, Y. Chen, Understanding influence power of opinion leaders in e-commerce networks: An opinion dynamics theory perspective. Information Sciences, 426(2018) 131-147.

[42]Y. Ren, D. Ji, Neural networks for deceptive opinion spam detection: An empirical study. Information Sciences, 385(2017)213-224.

[43]Y.C. Dong, S.H. Zhao, H.J. Zhang, F. Chiclana, E. Herrera-Viedma, A self-management mechanism for non-cooperative behaviors in large-scale group consensus reaching processes. IEEE Transactions on Fuzzy Systems, 26(6) (2018) 3276-3288.

[44]Y.C. Dong, Y.T. Liu, H.M. Liang, F. Chiclana, E. Herrera-Viedma, Strategic weight 
manipulation in multiple attribute decision making, Omega, 75 (2018) 154-164.

[45]Y.C. Dong, H.J. Zhang, E. Herrera-Viedma. Integrating experts' weights generated dynamically into the consensus reaching process and its applications in managing non-cooperative behaviors. Decision Support Systems, 84(2016) 1-15.

[46]R.A. Barrio, T. Govezensky, R. Dunbar, G. Iniguez, K. Kaski, Dynamics of deceptive interactions in social networks. Journal of the Royal Society Interface, 12(112) (2015) 463-474.

[47]G. Iñiguez, J. Török, T. Yasseri, K. Kaski, J. Kertész. Modeling social dynamics in a collaborative environment. EPJ Data Science, 3(1) (2014) 7.

[48]G. Iñiguez, T. Govezensky, R. Dunbar, K. Kaski, R.A. Barrio, Effects of deception in social networks. Proceedings of The Royal Society, 281(1790) (2014), 1-9.

[49]T. Gross, B. Blasius, Adaptive coevolutionary networks: A review. Journal of the Royal Society, 5 (2008) 259-271.

[50]M. Gupta, Consensus building process in group decision making - an adaptive procedure based on group dynamics, IEEE Transactions on Fuzzy Systems 26 (4) (2017) 1923-1933.

[51]N. Capuano, F. Chiclana, H. Fujita, E. Herrera-Viedma, V. Loia, Fuzzy group decision making with incomplete information guided by social influence, IEEE Transactions on Fuzzy Systems, 26 (3) (2018) 1704-1718. 